\title{
A high-performance scintillator-silicon-well X-ray microdetector based on DRIE techniques
}

\author{
J.G. Rocha ${ }^{*}$, J.H. Correia \\ Department of Industrial Electronics, University of Minho, Campus de Azurem, 4800 Guimaraes, Portugal
}

Accepted 18 January 2001

\begin{abstract}
This paper proposes an X-ray detector based on an array of scintillator crystals, CsI:Tl, encapsulated in a well that is etched into a silicon substrate by deep reactive ion etching. The X-ray energy is first converted to visible light which is detected by a photodetector in the well bottom. DRIE techniques are used to achieve perfect vertical side-walls $515 \mu \mathrm{m}$ deep, $100 \mu \mathrm{m}$ pixel square size and to maximize the fill factor. The inner walls of the well that are coated with aluminum for improving the number of photons which arrives to the detector and for reducing cross-talk between adjacent wells. Simulations and modeling show an improvement of approximately $26 \%$ in the detection efficiency using an aluminum layer. (C) 2001 Elsevier Science B.V. All rights reserved.
\end{abstract}

Keywords: Scintillator; Digital radiology; X-ray; DRIE; Micromachining

\section{Introduction}

The conventional X-ray imaging remains an analog technique while other medical imaging methods such as computed tomography, ultrasound and magnetic resonance imaging are digital. Digital radiography allows application of image processing techniques (e.g. detail improvement), application of sophisticated algorithms and real-time operation. Its requirements are sub-millimeter spatial resolution and good energy resolution. The application of X-ray imaging microdetectors in medical diagnostics is undeniably advantageous. Due to their compact size, wide dynamic range and digital data storage capacity, these imagers are very promising in the medical imaging technology when combined with readout microelectronics. A significant reduction of the dose of emitted radiation can also be achieved with these X-ray microdetectors [1].

This paper shows that an X-ray microdetector based on scintillator crystals on a silicon substrate is feasible and presents a good performance in order to increase the spatial resolution and to reduce the radiation dose applied to the patient.

\section{X-ray detection methods}

$\mathrm{X}$-rays can be converted in an electrical signal by means of several methods: the photodiode made in amorphous silicon, the photoconductor and the scintillator. Each method has its performance advantages and limitations on its use in practical X-ray imagers.

The simplest method is based on the photoelectric effect and consists of an array of photodiodes made in an amorphous silicon substrate. The X-rays that arrive are captured by the photodiodes where electron-hole pairs are produced. The amorphous silicon is preferred instead of crystalline silicon due to the fact that it presents an higher mechanical resistance when large area radiographic targets are made. An advantage of this method is that only $5 \mathrm{eV}$ of incoming energy is needed to produce a pair, but unfortunately, the Xray absorption of silicon is very low. A standard silicon wafer $(525 \mu \mathrm{m}$ thick) only absorbs about $2.2 \%$ of $100 \mathrm{keV}$ $\mathrm{X}$-ray energy. A layer $16.2 \mathrm{~mm}$ thick absorbs $50 \%$ of the same radiation (Fig. 1), so in a practical application, each photodiode needs to be $20 \mathrm{~mm}$ thick or more. This required thickness is completely impractical.

The photoconductor method uses photoconductive materials with higher absorption than silicon. These materials can be coated on an array of conductive charge collection plates each supplied with a storage capacitor. Currently, the best known commercial photoconductor, amorphous selenium, also has relatively low X-ray absorption and requires about

\footnotetext{
*Corresponding author. Tel.: +351-253-510190; fax: +351-253-510189.

E-mail address: gerardo@dei.uminho.pt (J.G. Rocha).
} 


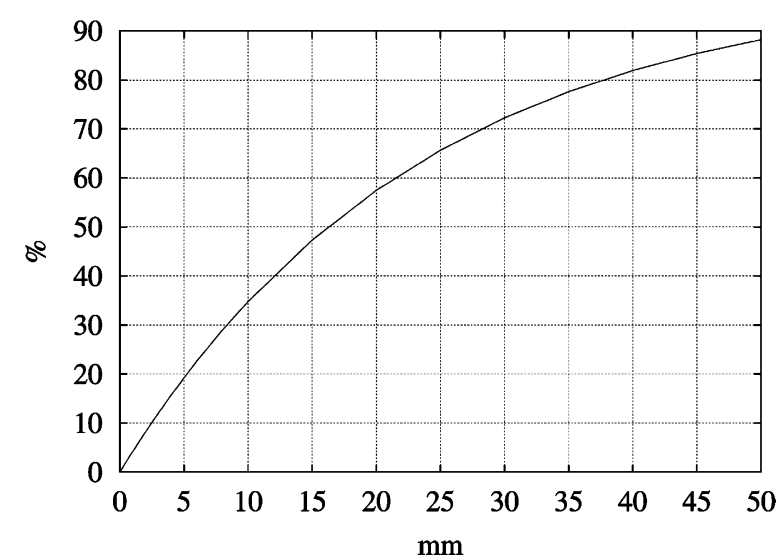

Fig. 1. Absorption rate of $100 \mathrm{keV} \mathrm{X}$-rays by a silicon wafer.

$50 \mathrm{eV}$ to produce an electron-hole pair. The main drawbacks are its high bias voltage which generates electrical insulation problems and its incompatibility with silicon technology. Another promising photoconductor material for X-ray imaging is the lead iodide $\left(\mathrm{PbI}_{2}\right)$, a wide bandgap semiconductor $\left(E_{\mathrm{g}}=2.3 \mathrm{eV}\right)$. It has a good X-ray absorption efficiency due to its high atomic number and good conversion efficiency [2]. The drawbacks are the previous mentioned for amorphous selenium.

The scintillator method uses a material that absorbs Xrays and converts its energy to visible light, easily detected by a photodiode. A good scintillator yields many visible light photons for each incoming X-ray. Usually consist of compounds formed by elements of high atomic number, which have high X-ray absorption.

The approach which uses scintillators seems to be the most feasible, due to the fact that the scintillator does not need bias voltage, its deposit process normally is simple and is an excellent $\mathrm{X}$-ray absorber.

\section{System design}

In medical imaging diagnosis, the $\mathrm{X}$-rays are produced with voltages from 25 to $120 \mathrm{kV}$ approximately. This produces an intensity peak ranging from 10 to $100 \mathrm{keV}$. In this project, an X-ray stimulable layer (scintillator crystal array) is used to convert $\mathrm{X}$-rays in visible light. The intensity of light is then converted to an electric signal by means of an array of photodiodes. The simplest method consists of the use of a phosphor scintillator above an array of photodiodes as is shown in Fig. 2(a). The drawback is that the blur diameter on image is comparable to the scintillator thickness, i.e. the thicker the scintillator (desirable to increase the quantum absorption efficiency) the blurrier the image will be.

In order to reduce the blur of the output image two approaches are used:

- The first one uses the cesium iodide in a columnar structure $[3,4]$, when deposited under very specific conditions [5]. However, due to the cross-talk between the cesium iodide columns, the spatial resolution is still limited.

- The second one uses microcavities made in a silicon substrate, which are filled with the scintillator [6,7]. The problem of this approach is that CsI has a low refractive index $(\approx 1.8)$ at $560 \mathrm{~nm}$ when compared with silicon $(\approx 4)$. Only the light produced by the scintillator that reaches the silicon wall at low angles is reflected. The remainder light is absorbed by the silicon walls or transmitted to the adjacent wells.

In order to reduce the blur and increase the efficiency, this paper follows the second method, but uses the aluminum coating to reflect the visible light produced by the scintillator (Fig. 2(b)). Moreover, introducing a reflective layer above the scintillator (in the X-ray path) confines the light inside of the well increasing the efficiency. Thus, the device consists in an array of micromachined silicon wells (Figs. 3 and 4). The inner walls of the wells are coated by an aluminum layer which is deposited by means of selective electroplating. Aluminum has a better performance than other metals (e.g. silver and gold) due to its low density and consequently low X-ray absorption. Moreover, aluminum is a siliconcompatible material. The scintillator is deposited below the

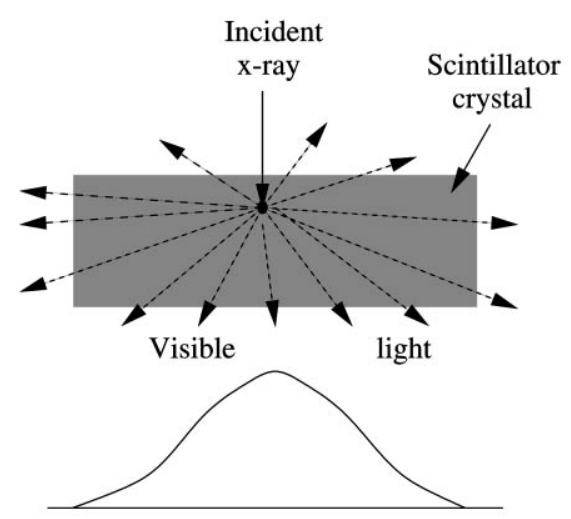

(a)

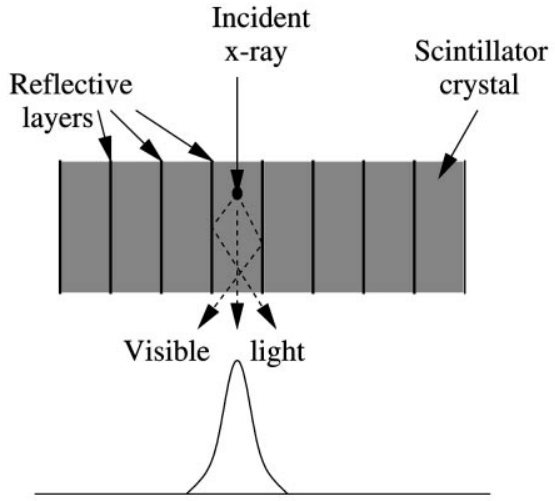

(b)

Fig. 2. Effect of reflective layer on the spatial resolution: (a) a single scintillator layer; (b) structured columnar array. 


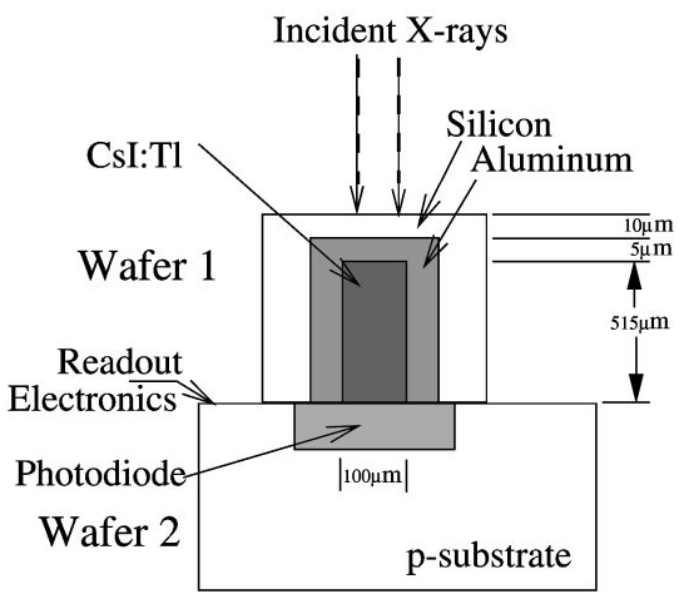

Fig. 3. A complete well in cross-section (not to scale).

aluminum layer. The X-rays penetrate the silicon and aluminum top walls and hit the scintillator which produces visible light (wavelength near $560 \mathrm{~nm}$ for CsI:Tl). The electroplated inner walls of the well allow multiple reflections and guides almost all produced light to the photodiodes. A first prototype is being fabricated at TuDelft, The Netherlands.

\section{Scintillator materials}

A large number of materials have been found to emit light, when ionizing radiation falls upon them. The most widely employed scintillators include inorganic-based liquids and plastics. The inorganic-based liquids and plastics tend to have the best light output and linearity, but are known to be slow in their response times, whereas organic scintillators are faster but yield less light. At present the industry

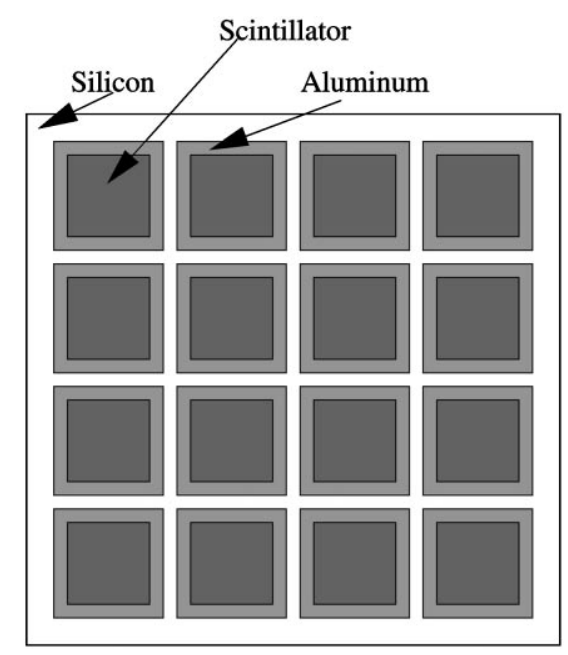

Fig. 4. A silicon well array filled with scintillators (bottom view of wafer 1).
Table 1

Light yield by some scintillator crystals at room temperature

\begin{tabular}{lll}
\hline Crystal & $L_{\mathrm{y}}$ (photons/MeV) & $\lambda(\mathrm{nm})$ \\
\hline $\mathrm{NaI}: \mathrm{Tl}$ & 38000 & 303 \\
$\mathrm{CsI}: \mathrm{Tl}$ & 59000 & 560 \\
$\mathrm{CsI}: \mathrm{Na}$ & 39000 & 420 \\
$\mathrm{Lu}_{2} \mathrm{SiO}_{5}: \mathrm{Ce}$ & 30000 & 420 \\
$\mathrm{Y}_{2} \mathrm{SiO}_{5}: \mathrm{Ce}$ & 45000 & 420 \\
$\mathrm{Y}_{3} \mathrm{Al}_{5} \mathrm{O}_{12}: \mathrm{Ce}$ & 19700 & 390 \\
$\mathrm{ZnS}_{\mathrm{Ang}}$ & 49000 & 450 \\
$\mathrm{CaI}_{2}$ & 86000 & 410 \\
\hline
\end{tabular}

routinely produces about two dozen types of inorganic crystalline scintillators. Thin layers of most inorganic scintillators effectively absorb X-rays quanta in the energy region $1-100 \mathrm{keV}$. The main requirement of scintillators for these energies is a high light output (photons/MeV). Table 1 presents a group of scintillators with the highest light outputs at room temperature [8]. Very high scintillation efficiency $\eta=0.25$ has been found for $\mathrm{CaI}_{2}$ and $\mathrm{CaI}_{2}: \mathrm{Eu}$ crystals [9]. The scintillators based on $\mathrm{CaI}_{2}$ are not used in practical applications because they are highly hygroscopic and easily cleaveable.

The most used scintillators are CsI:Tl and NaI:Tl because of their high light yield and reasonably fast decay time and most importantly because of the low costs to grow these materials. CsI has quite a high X-ray absorption coefficient and is less susceptible to thermal and mechanical shock than sodium iodide (NaI). CsI:Tl is also flexible, which permits shaping to a certain extent without fracture. One of the most important characteristics of cesium iodide is that it emits visible light $560 \mathrm{~nm}$ wavelength when doped with 0.02 $0.03 \%$ of thallium ions. The combination of CsI:Tl and amorphous silicon has one of the highest quantum efficiencies of all used materials. Moreover, CsI:Tl has the peak of light output at room temperature (see Fig. 5). As a drawback (being less hygroscopic than NaI:Tl), CsI:Tl is a good water absorber and must be protected against humidity.

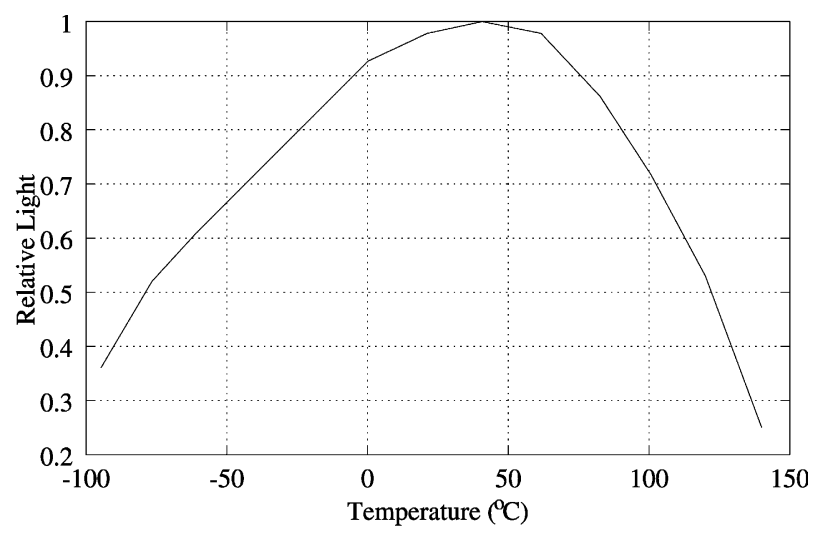

Fig. 5. Relative light output of CsI:Tl as a function of temperature. 


\section{Efficiency per well analysis}

The ratio between the total area of the scintillator surface $(A)$, and the area seen by the photodiode (a) (Figs. 3 and 4), is defined as

$R_{\mathrm{A}}=\frac{a}{A}$.

The light that reaches the photodiode, without reflections and considering that the scintillator does not absorb its own light, is

$L_{\mathrm{pd}}^{*}=L_{\mathrm{y}} R_{\mathrm{A}}$,

where $L_{\mathrm{y}}$ is the total light yield by the scintillator.

If the scintillator surface is coated by some material, except at the photodiode side, the light that reaches it is

$L_{\mathrm{cm}}=L_{\mathrm{y}}-L_{\mathrm{pd}}^{*}=L_{\mathrm{y}}\left(1-R_{\mathrm{A}}\right)$.

At the interface with the coating material, a percentage of the light is lost and the remaining one is reflected back to the scintillator. The percentage of losses can be defined as

$R_{\text {loss }}=\frac{L_{1}}{L_{\mathrm{cm}}}$,

where $L_{1}$ is the total light lost.

After multiple reflections and losses, the light that reaches the photodiode can be calculated by the geometric series

$\sum_{i=0}^{\infty} L_{\mathrm{y}} R_{\mathrm{A}}\left[\left(1-R_{\mathrm{A}}\right)\left(1-R_{\text {loss }}\right)\right]^{i}$.

Once $\left[\left(1-R_{\mathrm{A}}\right)\left(1-R_{\text {loss }}\right)\right]<1$, the series is convergent, and the total light that reaches the photodiode is

$L_{\mathrm{pd}}=\frac{L_{\mathrm{y}} R_{\mathrm{A}}}{1-\left(1-R_{\mathrm{A}}\right)\left(1-R_{\text {loss }}\right)}$.

The total area of scintillator surface is $0.224 \mathrm{~mm}^{2}$ and the area seen by the photodiode is $0.01 \mathrm{~mm}^{2}$. The ratio between both areas is $R_{\mathrm{A}}=0.0446$. If the electroplated aluminum layer has $R_{\text {loss }} \simeq 3 \%$, Eq. (6) says that the total light that reaches the photodiode is approximately $0.6 L_{\mathrm{y}}$. Without the aluminum layer, the interface between the scintillator and the silicon walls reflects about $85 \%$ of incident radiation, due to its high refractive index relatively to CsI, and $R_{\text {loss }} \simeq 0.15$. In this case, the total light collected by the photodiode is $0.23 L_{\mathrm{y}}$.

Considering that the absorption of X-rays by the aluminum, at low energies approximates $11 \%$ the improvement achieved is $26 \%$. At high energy, the improvement increases because at high $\mathrm{X}$-ray energy, the aluminum absorbs less radiation.

\section{Cross-talk between adjacent detectors}

The electroplated inner walls of the well avoid that the light produced by one scintillator crystal can be detected by

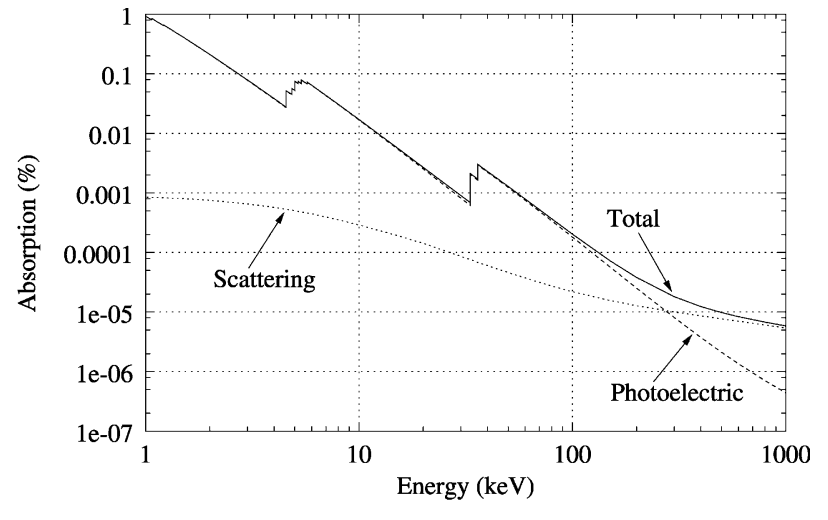

Fig. 6. Partial interaction coefficients of X-rays with CsI.

adjacent photodiodes. The cross-talk problem can occur due to the scattering (Rayleigh and Compton) of incident X-rays when interact with the scintillator. Fig. 6 shows the total energy absorbed by the scintillator and the percentages absorbed by photoelectric effect and scattered. At $10 \mathrm{keV}$ the scattering effect occurs only with $1.7 \%$ of incident radiation and increases with the radiation energy. At $100 \mathrm{keV}$, the scattering is about $10.7 \%$ of absorbed radiation by photoelectric effect, value that begins to be unacceptable once the scattered X-ray photons can produce light in the neighbor scintillators.

\section{Simulation results}

The simulation results are based on the mass energytransfer and mass energy-absorption coefficients of the materials [10]. The software was developed by the microtechnologies lab, in the Industrial Electronics Department of University of Minho.

Fig. 7 shows the incident $\mathrm{X}$-rays rate that go through the silicon and aluminum walls, as a function of the radiation energy and the thickness of the aluminum layer. As it can be seen on the graphic, for a wall thickness of about $15 \mu \mathrm{m}$ ( $10 \mu \mathrm{m}$ of silicon and $5 \mu \mathrm{m}$ of aluminum), $89 \%$ of $10 \mathrm{keV}$ incident radiation goes through the wall.

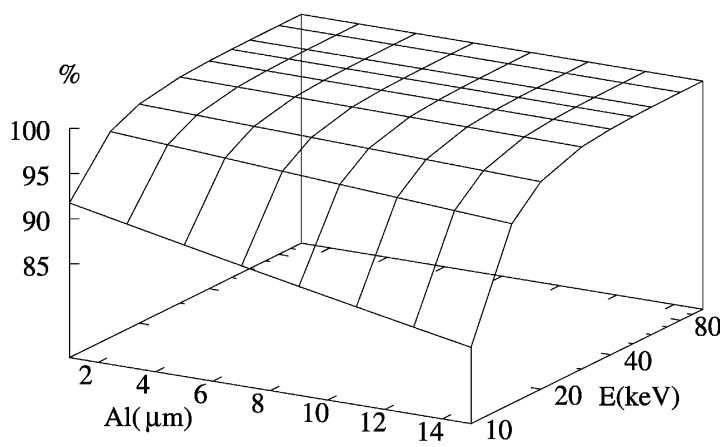

Fig. 7. Energy transmitted by a layer stack composed by $10 \mu \mathrm{m}$ of silicon and $1-15 \mu \mathrm{m}$ of aluminum. 


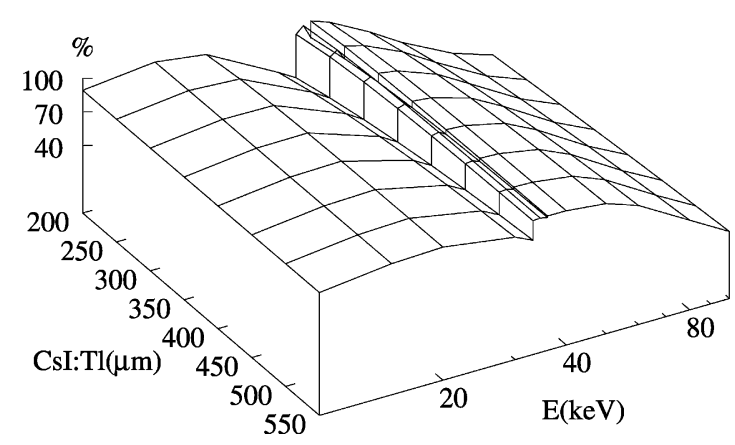

Fig. 8. Energy rate absorbed by the scintillator as a function of its thickness and X-ray energy.

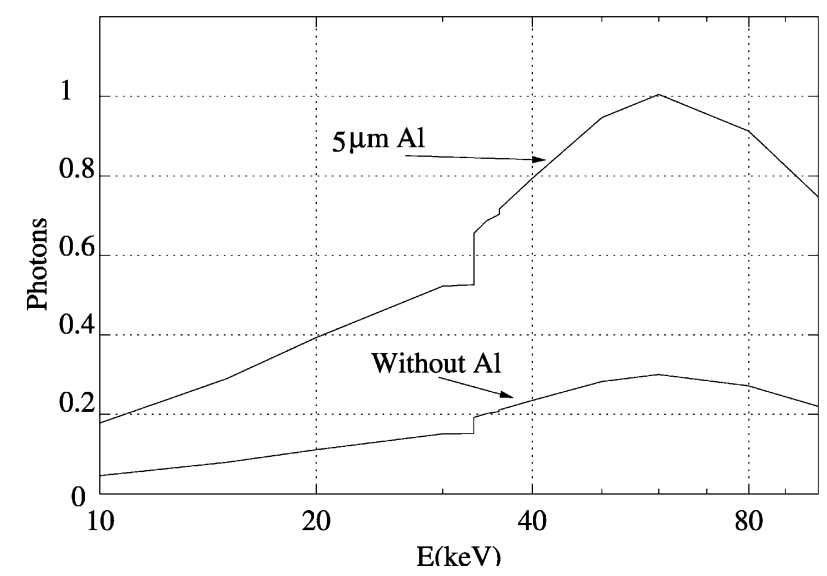

Fig. 9. Number of photons that reach the photodiode (normalized to the high-intensity peak in the $5 \mu \mathrm{m} \mathrm{Al}$ layer).

Fig. 8 shows the rate of X-rays which is absorbed by each scintillator crystal, regarding the effect of the top walls. It can be seen in the same figure that for the $40 \mathrm{keV}$ X-rays, about $99 \%$ of them are absorbed by a $510 \mu \mathrm{m}$ scintillator.

Fig. 9 shows the number of photons that reach the photodiode, for a scintillator thickness of $510 \mu \mathrm{m}$ and walls with $10 \mu \mathrm{m}$ of silicon. The X-ray source is powered with 20 $120 \mathrm{kV}, 500 \mathrm{~mA}$ and the exposure time is about $10 \mathrm{~ms}$. The maximum efficiency is reached near $60 \mathrm{keV}$ with this setup. The quantum efficiency at photon energies below $60 \mathrm{keV}$ can be increased lowering the thickness of the topwall well (e.g. reducing the silicon or aluminum layers). The quantum efficiency increases with the scintillator thickness at energies above $60 \mathrm{keV}$.

\section{Conclusions}

This approach with structured columnar scintillator and high-reflective inner walls has better performance than the classical method (only one powder phosphor screen [11]). Moreover, the electroplating of the well walls with a highreflective layer avoids cross-section problems and decrease losses. Hexagonal-well shape is being studied in order to increase detection efficiency and the fill factor.

\section{Acknowledgements}

The authors wish to acknowledge the cooperation of Professor R.F. Wolffenbuttel's group from the Laboratory for Electronic Instrumentation, TuDelft, The Netherlands. This work was supported by the Foundation of Science and Technology, Portugal (FCT-33751/99).

\section{References}

[1] J.P. Heyne, H. Merbold, J. Sehner, R. Newmann, M.G. Freesmeyer, L.J. Mentzel, W.A. Kaiser, Reduction of radiation dose by using digital luminescence radiography compared to conventional screen film system with grid cassette, in: Proceedings of the ECR-99, March 1999.

[2] K.S. Shah, P. Bennett, L. Cirignano, Y. Dmitriyev, M. Klugerman, K. Mandal, L.P. Moy, X-ray imaging with semiconductor films, in: Proceedings of the SPIE Conference, San Diego, CA, July 1998, pp. 102-113.

[3] I. Fujieda, G. Cho, J. Drewery, T. Gee, T. Jing, S.N. Kaplan, V. Perez Mendez, D. Wildermuth, X-ray and charged particle detection with $\mathrm{CsI}(\mathrm{Tl})$ layer coupled to a $\alpha$-Si:H photodiode layers, IEEE Trans. Nucl. Sci. 38 (2) (April 1991) 255-262.

[4] J. Alain, L. Lapierre, A. Legros, H. Mani, Z. Shukri, H. Rougeot, New cesium iodide-selenium X-ray detector structure for digital radiography and fluoroscopy, Proc. SPIE 3659 (May 1999) 298-306.

[5] J.P. Galves, J.M. Gutierrez, Process for producing a scintillator screen, US Patent 4,287,230 (September 1981).

[6] P. Kleimann, J. Linnros, C. Fröjdh, C.S. Petersson, An X-ray imaging pixel detector based on a scintillating guides screen, IEEE Trans. Nucl. Sci. 47 (4) (August 2000) 1483-1486.

[7] C.P. Allier, R.W. Hollander, P.M. Sarro, M. de Boer, C.W.E. van Eijk, Scintillation light read-out by low-gain thin avalanche photodiodes in silicon wells, IEEE Trans. Nucl. Sci. 47 (4) (August 2000) 1303-1306.

[8] S.E. Derenzo, W.W. Moses, Experimental efforts and results in finding new heavy scintillators, in: Proceedings of the "Crystal 2000" International Workshop on Heavy Scintillators for Scientific and industrial Applications, France, 1993.

[9] P.A. Rodnyi, Physical processes in inorganic scintillators, CRC Press, Boca Raton, 1997.

[10] S.M. Seltzer, Calculation of photon mass energy-transfer and mass energy-absorption coefficients, Rad. Res. 136 (1993) 147-170.

[11] J.A. Rowlands, Current advances and future trends in X-ray digital detectors, IEEE Instrum. Meas. Mag. December (1998) 26-28.

\section{Biographies}

Josè Gerardo V. Rocha graduated in industrial electronics engineering in 1995 and obtained his MSc degree in 1999 at the University of Minho, Portugal. Since 1996 he has been a lecturer in the Department of Industrial Electronics, University of Minho, Portugal, and he is involved in the research of radiation sensors.

Josè Higino Correia graduated in physical engineering at University of Coimbra, Portugal, in 1990. He obtained his PAPCC (equivalent to M.Sc degree) in 1994 at University of Minho, Portugal, and his Ph.D degree in 1999 at Delft University of Technology, The Netherlands. His thesis work dealt with the development of optical microsystems in silicon for the visible part of the spectrum. Since May 1999 he has been an assistant professor at the Department of Industrial Electronics of the University of Minho and he is involved in instrumentation and measurement in general and on-chip functional integration of microelectronic circuits and silicon sensors. 